\title{
optica
}

\section{What does it take to detect entanglement with the human eye?: supplemental material}

\author{
Valentina Caprara Vivoli ${ }^{1,2}$, Pavel Sekatski ${ }^{3}$, and Nicolas Sangouard ${ }^{4}$ \\ ${ }^{1}$ Group of Applied Physics, University of Geneva, CH-1211 Geneva 4, Switzerland \\ ${ }^{2}$ QuTech, Delft University of Technology, Lorentzweg 1, 2611 CJ Delft, Netherlands \\ ${ }^{3}$ Institut for Theoretische Physik, Universitat of Innsbruck, Technikerstraße 25, A-6020 Innsbruck, Austria \\ ${ }^{4}$ Department of Physics, University of Basel, Klingelbergstrasse 82, 4056 Basel, Switzerland
}

Published 3 May 2016

This document provides supplementary information to "What does it take to detect entanglement with the human eye?," http://dx.doi.org/10.1364/optica.3.000473.

(C) 2016 Optical Society of America

\section{SUPPLEMENTAL MATERIAL I}

In this section, we provide a convenient expression for a threshold detector with non-unit efficiency (threshold $\theta$ and efficiency $\eta)$ and for the same detector upgraded with a displacement $D(\alpha)=e^{\alpha a^{+}-\alpha^{*} a}$. In the ideal case $\eta=1$, the no-click event can be identified with $P_{\mathrm{ns}}^{\theta, \eta=1}=\sum_{m=0}^{\theta-1}|m\rangle\langle m|$. To model nonunit efficiency, we can use a beamsplitter interaction $U_{B S}$ between the detected mode $\mathrm{A}$ and an empty ancillary mode $\mathrm{C}$, with bosonic operators $a, a^{\dagger}$, and $c, c^{\dagger}$, respectively. The beam splitter operator can thus be written as [1] $U_{B S}|0\rangle_{c}=C_{L}=$ $e^{\tan \gamma a c^{\dagger}} e^{\ln (\cos \gamma) a^{\dagger} a}|0\rangle_{c}$, where $\cos ^{2} \gamma=\eta$. The no-click event operator can be written as

$$
P_{\mathrm{ns}}^{\theta, \eta}=\operatorname{tr}_{C} U_{B S}^{\dagger} P_{\mathrm{ns}}^{\theta, 1} U_{B S}|0\rangle\left\langle\left. 0\right|_{C}=C_{L}^{\dagger} \sum_{m=0}^{\theta-1} \mid m\right\rangle\langle m| C_{L} .
$$

Noting that $|m\rangle\langle m|=\delta_{a^{+} a, m}$ and using $\delta_{n, m}=\lim _{z \rightarrow 0_{+}} \frac{1}{m !} \frac{d^{m}}{d z^{m}} z^{n}$, we have

$$
P_{\mathrm{ns}}^{\theta, \eta}=\sum_{m=0}^{\theta-1} \lim _{z \rightarrow 0_{+}} \frac{1}{m !} \frac{d^{m}}{d z^{m}} C_{L}^{\dagger} z^{a^{+} a} C_{L}
$$

Together with $C_{L}^{+} z^{a^{\dagger} a} C_{L}=(1-\eta+z \eta)^{a^{\dagger} a}[1]$, we find

$$
\begin{array}{r}
P_{\mathrm{ns}}^{\theta, \eta}=\sum_{m=0}^{\theta-1} \lim _{z \rightarrow 0_{+}} \frac{1}{m !} \frac{d^{m}}{d z^{m}}(1-\eta+z \eta)^{a^{+} a} \\
=\sum_{m=0}^{\theta-1} \frac{\eta^{m}}{m !} \lim _{z \rightarrow 0_{+}} \frac{d^{m}}{d(1-\eta+z \eta)^{m}}(1-\eta+z \eta)^{a^{+} a}
\end{array}
$$

which simplifies to

$$
\begin{aligned}
P_{\mathrm{ns}}^{\theta, \eta} & =\sum_{m=0}^{\theta-1} \frac{\eta^{m}}{m !} \frac{d^{m}}{d(1-\eta)^{m}}(1-\eta)^{a^{+} a} \\
& =\frac{\eta^{\theta}}{(\theta-1) !} \frac{d^{\theta-1}}{d(1-\eta)^{\theta-1}}\left[\frac{(1-\eta)^{a^{+} a}}{\eta}\right],
\end{aligned}
$$

where $\frac{d^{m}}{d(1-\eta)^{m}}$ is the $\mathrm{m}$-th derivative with respect to $(1-\eta)$. The click event can be deduced from $P_{\mathrm{s}}^{\theta, \eta}=\mathbb{1}-P_{\mathrm{ns}}^{\theta, \eta}$.

From the formula Eq. (S.5) it is easy to obtain a similar expression for the detecor upgraded with a displacement. One simply uses $D(\alpha)^{\dagger} a D(\alpha)=a+\alpha$ and obtains

$$
\begin{aligned}
D(\alpha)^{\dagger} P_{\mathrm{ns}}^{\theta, \eta} D(\alpha) & =\frac{\eta^{\theta}}{(\theta-1) !} \frac{d^{\theta-1}}{d(1-\eta)^{\theta-1}}\left[\frac{(1-\eta)^{\left(a^{+}+\alpha^{*}\right)(a+\alpha)}}{\eta}\right] \\
& =\frac{\eta^{\theta}}{(\theta-1) !} \frac{d^{\theta-1}}{d(1-\eta)^{\theta-1}}\left[\frac{: e^{-\eta\left(a^{+}+\alpha^{*}\right)(a+\alpha)}:}{\eta}\right],
\end{aligned}
$$

where we used $(1-\eta)^{a^{+} a}=: e^{-\eta a^{+} a}:$ [4] in the second equation (: : denotes the ordering where all the annihilation operators are on the right). Let us illustrate the usefulness of the last formula on the two cases which will be important for later: a coherent state $|\beta\rangle$ and a Fock state $|\ell\rangle$.

For the coherent state, Eq. (S.6) directly leads to

$$
\left\langle\beta\left|D(\alpha)^{\dagger} P_{\mathrm{ns}}^{\theta, \eta} D(\alpha)\right| \beta\right\rangle=\frac{\eta^{\theta}}{(\theta-1) !} \frac{d^{\theta-1}}{d(1-\eta)^{\theta-1}}\left[\frac{e^{-\eta|\alpha+\beta|^{2}}}{\eta}\right] .
$$


Eq. (S.7) is useful to compute the no-click probability from the $P$-representation of any state. In particular, we will use it to evaluate the value taken by the witness in the setup presented in Fig. 2 of the main text with a source based on spontaneous parametric down conversion, see Sec. 5 .

In the case of the Fock state, the calculation is a little bit more evolved. Note first that one can re-write the operator of Eq. (S.6) as : $e^{-\eta\left(a^{\dagger} a+\alpha^{*} a+\alpha a\right)}:=e^{-\eta \alpha a^{\dagger}}: e^{-\eta a^{\dagger} a}: e^{-\eta \alpha^{*} a}=$ $e^{-\eta \alpha a^{\dagger}}(1-\eta)^{a^{\dagger} a} e^{-\eta \alpha^{*} a}$. For any two Fock states $|k\rangle$ and $|\ell\rangle$, this leads to

$$
\left\langle k\left|D(\alpha)^{\dagger} P_{\mathrm{ns}}^{\theta, \eta} D(\alpha)\right| \ell\right\rangle=\frac{\eta^{\theta}}{(\theta-1) !} \frac{d^{\theta-1}}{d(1-\eta)^{\theta-1}}\left[\frac{e^{-\eta|\alpha|^{2}} p_{k, \ell}^{\alpha}(\eta)}{\eta}\right],
$$

where $p_{k, \ell}^{\alpha}(\eta)=\left\langle k\left|e^{-\eta \alpha a^{\dagger}}(1-\eta)^{a^{\dagger} a} e^{-\eta \alpha^{*} a}\right| \ell\right\rangle$ is a polynimial in $\eta$ of degree $k+\ell$ that is straightforward to compute. We will use Eq. (S.8) for the construction of our entanglement witness in Sec. 2 and 4, and to compute the probability of the event "not seen" for different Fock states in Sec. 3 (some cases are plotted in Fig. S1).

\section{SUPPLEMENTAL MATERIAL II}

Here we give details on how the entanglement witness has been derived, assuming first that Alice and Bob both have qubits.

We recall that the proposed entanglement witness has the following form (see main text)

$$
W=\int_{0}^{2 \pi} \frac{d \varphi}{2 \pi} U_{\varphi}^{\dagger} \otimes U_{\varphi}^{\dagger}\left(\sigma_{\alpha}^{1} \otimes \sigma_{\beta}^{7}\right) U_{\varphi} \otimes U_{\varphi}
$$

where $\sigma_{\alpha}^{\theta}=D(\alpha)^{\dagger}\left(2 P_{\text {ns }}^{\theta, 1}-\mathbb{1}\right) D(\alpha)$ is the observable obtained when the value -1 is given in case of a "click"("seen") event and the +1 is given otherwise. $U_{\varphi}=e^{i \varphi a^{\dagger} a}$ is the phase rotation of mode A (similarly for B). For simplicity, the non-unit efficiency of the detectors are seen as loss operating on the state. This allows one to focus on the construction of an entanglement witness with unit-efficiency detectors. Now consider the restriction of the witness (S.9) to the qubit case, i.e. to the Hilbert space spanned by the vectors $\{|00\rangle,|10\rangle,|01\rangle,|11\rangle\}$ where Alice and Bob have no more than one photon each. Note right away that the coherences associated to terms with different total photon numbers (such as $|00\rangle\langle 10|$ ) do not contribute due to the phase averaging. The entanglement witness operator, expressed in the same basis, thus has the block-diagonal structure

$$
W=\left(\begin{array}{cccc}
W_{00} & & & \\
& W_{10} & W_{c} & \\
& W_{c}^{*} & W_{01} & \\
& & & W_{11}
\end{array}\right) .
$$

In other words, The block-diagonal structure is ensured by the fact that our measurement setup is invariant under global phase change. Hence no coherence between sectors with different total photon number can be observed (this is the superselection rule implied by the global phase symmetry). In Eq. (S.10) each element depends on the displacements $\alpha, \beta$, and the threshold $\theta$. Their explicite expression can be directly obtained by plugging Eq. (S.8) into Eq. (S.9) and setting $\eta \rightarrow 1$.
Now consider a general density matrix $\rho$ over the same Hilbert space. We look for the maximal value that $\langle W\rangle=\operatorname{tr}(W \rho)$ can take over the set of separable states, i.e. over the states $\rho$ that stay positive under partial transposition (for two qubits the two conditions are equivalent [2]). In summary, we want to optimize $\langle W\rangle$ over the states $\rho(4 \times 4$ Hermitian matrices $)$ such that

1. $\rho \geq 0$,

2. $\operatorname{tr}(\rho)=1$,

3. $\rho^{T_{b}} \geq 0$.

Here $\rho^{T_{b}}$ stands for the partial transposition over one party. Due to the simple block-diagonal structure of $W$, it is straightforward to show that for any separable state $\rho$ with photon number statistics $p_{i j}=\langle i j|\rho| i j\rangle$

$$
\operatorname{tr}(W \rho) \leq W_{\mathrm{ppt}}=\sum_{i, j=0}^{1} W_{i j} p_{i j}+2\left|W_{c}\right| \sqrt{p_{00} p_{11}}
$$

Hence, any state $\rho_{\exp }$ for which

$$
\operatorname{tr}\left(\rho_{\exp } W\right)-W_{\mathrm{ppt}}>0
$$

has a negative partial transpose, i.e. is necessarily entangled. It is important to stress that $W_{\text {ppt }}$ requires the knowledge of the photon number statistics $\vec{p}=\left\{p_{i j}\right\}$ only. The elements $W_{i j}=\langle i j|W| i j\rangle$ and $\left|W_{c}\right|=|\langle 10|W| 01\rangle|$ are computed by combining Eq. (S.9) with Eq. (S.8). We show in the next section how the $p_{i j}$ s can be bounded.

\section{SUPPLEMENTAL MATERIAL III}

Figure S1 shows the probability of having no click on a threshold detector $(\theta=7)$ with a number state $|n\rangle$ that is displaced in phase space as a function of the displacement amplitude $\beta$, $\left.\left(P_{B}(+1|\beta| n\rangle,\right)=\left\langle n\left|D^{\dagger}(\beta) P_{\text {ns }}^{7,1} D(\beta)\right| n\right\rangle\right)$ for $n=0$ (blue full line), 1 (purple dashed line), 2 (brown dash-dotted line), 3 (green dotted line). We show how to bound $p_{00}, p_{10}, p_{01}$, and $p_{11}$ from these results.

In what follows, we call $p_{0 n}=\left\langle 0 n\left|\rho_{\exp }\right| 0 n\right\rangle$, the projection of $\rho_{\exp }$ in $|0 n\rangle_{A B}, p_{0 A}=P_{A}\left(+1 \mid 0, \rho_{\exp }\right)=\operatorname{tr}\left(\rho_{\exp } P_{n S}^{1,1} \otimes \mathbb{1}\right)=$ $\operatorname{tr}\left(\rho_{\exp }|0\rangle_{A}\langle 0| \otimes \mathbb{1}_{B}\right)$ the probability of having 0 photon in mode $\mathrm{A}$, and $P_{A}\left(-1 \mid 0, \rho_{\exp }\right)=1-\operatorname{tr}\left(\rho_{\exp }|0\rangle_{A}\langle 0| \otimes \mathbb{1}_{B}\right)$ the probability of having more than 0 photon in $A$. In order to bound $p_{00}$ and $p_{10}$, we consider the displacement amplitude $\beta_{0}(\sim 2.71)$ such that $\left.\left.P_{B}\left(+1\left|\beta_{0},\right| 0\right\rangle\right)=P_{B}\left(+1\left|\beta_{0},\right| 2\right\rangle\right)$ and $\left.\left.P_{B}\left(+1\left|\beta_{0},\right| n \neq 1\right\rangle\right)<P_{B}\left(+1\left|\beta_{0},\right| 1\right\rangle\right)$. Using $\sum_{n=0}^{\infty}|n\rangle\langle n|=\mathbb{I}$, we have

$$
\begin{aligned}
P_{A B}\left(+1+1 \mid 0 \beta_{0}, \rho_{\exp }\right)= & \sum_{n=0}^{+\infty}\left\langle 0 n\left|\rho_{\exp }\right| 0 n\right\rangle \\
\quad \times\left\langle n\left|D^{\dagger}\left(\beta_{0}\right) P_{\mathrm{ns}}^{7,1} D\left(\beta_{0}\right)\right| n\right\rangle & \\
= & \left.\sum_{n=0}^{+\infty} p_{0 n} P_{B}\left(+1\left|\beta_{0},\right| n\right\rangle\right) .
\end{aligned}
$$

Using $\left.\left.P_{B}\left(+1\left|\beta_{0},\right| n \neq 1\right\rangle\right)<P_{B}\left(+1\left|\beta_{0},\right| 1\right\rangle\right)$, one can manipulate 


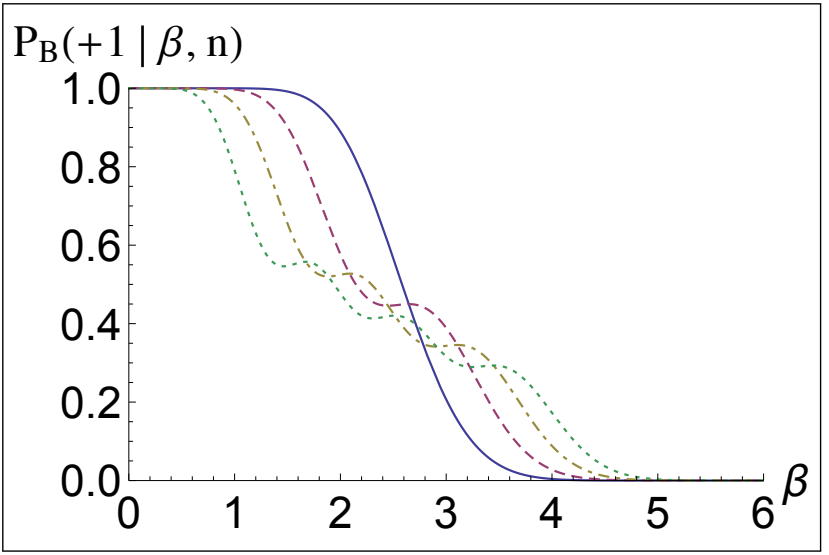

Fig. S1. Probability $\left.P_{B}(+1|\beta| n\rangle,\right)$ for having no click on a threshold detector $(\theta=7)$ with a number state $|n\rangle$ (for $n=0$ (blue full line), 1 (purple dashed line), 2 (brown dash-dotted line), 3 (green dotted line)) that is displaced in phase space as a function of the displacement amplitude $\beta$

the previous expression in the following way

$$
\begin{aligned}
\left.\sum_{n=0}^{+\infty} p_{0 n} P_{B}\left(+1\left|\beta_{0},\right| n\right\rangle\right) \leq p_{00} P_{B}( & \left.\left.+1\left|\beta_{0},\right| 0\right\rangle\right) \\
& \left.+\sum_{n=1}^{+\infty} p_{0 n} P_{B}\left(+1\left|\beta_{0},\right| 1\right\rangle\right) \\
=\left(P_{B}(\right. & \left.\left.\left.\left.+1\left|\beta_{0},\right| 0\right\rangle\right)-P_{B}\left(+1\left|\beta_{0},\right| 1\right\rangle\right)\right) p_{00} \\
& \left.+P_{B}\left(+1\left|\beta_{0},\right| 1\right\rangle\right) p_{0 A} .
\end{aligned}
$$

This leads to the upperbound

$$
p_{00} \leq \underbrace{\frac{\left.P_{A B}\left(+1+1 \mid 0 \beta_{0}, \rho_{\exp }\right)-P_{B}\left(+1\left|\beta_{0},\right| 1\right\rangle\right) P_{A}\left(+1 \mid 0, \rho_{\exp }\right)}{\left.\left.P_{B}\left(+1\left|\beta_{0},\right| 0\right\rangle\right)-P_{B}\left(+1\left|\beta_{0},\right| 1\right\rangle\right)}}_{\bar{p}_{00}} .
$$

In the same way, replacing the outcome +1 (no-click) with -1 (click) for the mode $\mathrm{A}$, we get

$$
\begin{aligned}
P_{A B}\left(-1+1 \mid 0 \beta_{0}, \rho_{\exp }\right)= & \sum_{\substack{n=0 \\
m=1}}^{+\infty}\left\langle m n\left|\rho_{\exp }\right| m n\right\rangle \\
& \quad \times\left\langle n\left|D^{\dagger}\left(\beta_{0}\right) P_{\mathrm{ns}}^{7,1} D\left(\beta_{0}\right)\right| n\right\rangle \\
\leq & \left.\left.\left(P_{B}\left(+1\left|\beta_{0},\right| 0\right\rangle\right)-P_{B}\left(+1\left|\beta_{0},\right| 1\right\rangle\right)\right) \\
& \quad \times \sum_{m \geq 1} p_{m 0} \\
& \left.+P_{B}\left(+1\left|\beta_{0},\right| 1\right\rangle\right) P_{A}\left(-1 \mid 0, \rho_{\exp }\right) .
\end{aligned}
$$

This leads to the following upperbound for $p_{10}$

$$
\begin{aligned}
p_{10} & \leq \sum_{m \geq 1} p_{m 0} \\
& \leq \underbrace{\frac{\left.P_{A B}\left(-1+1 \mid 0 \beta_{0}, \rho_{\exp }\right)-P_{B}\left(+1\left|\beta_{0},\right| 1\right\rangle\right) P_{A}\left(-1 \mid 0, \rho_{\exp }\right)}{\left.\left.P_{B}\left(+1\left|\beta_{0},\right| 0\right\rangle\right)-P_{B}\left(+1\left|\beta_{0},\right| 1\right\rangle\right)}}_{\bar{p}_{10}} .
\end{aligned}
$$

To bound $p_{01}$ and $p_{11}$ we consider the displacement amplitude $\beta_{1}(\sim 2.09)$ such that $\left.\left.P_{B}\left(+1\left|\beta_{1},\right| 1\right\rangle\right)=P_{B}\left(+1\left|\beta_{1},\right| 2\right\rangle\right)$ (we also have $\left.P_{B}\left(+1\left|\beta_{1},\right| 0\right\rangle\right) \geq P_{B}\left(+1\left|\beta_{1},\right| n \geq 1\right\rangle$ ) and $\left.\left.P_{B}\left(+1\left|\beta_{1},\right| n \geq 3\right\rangle\right) \leq P_{B}\left(+1\left|\beta_{1},\right| 1\right\rangle\right)$ for the same displacement amplitude). Following the same line of thought used for $p_{00}$ and $p_{01}$, we get

$$
\begin{gathered}
p_{01} \leq \underbrace{\frac{\left.P_{A B}\left(+1+1 \mid 0 \beta_{1}, \rho_{\exp }\right)-P_{B}\left(+1\left|\beta_{1},\right| 0\right\rangle\right) P_{A}\left(+1 \mid 0, \rho_{\exp }\right)}{\left.\left.P_{B}\left(+1\left|\beta_{1},\right| 1\right\rangle\right)-P_{B}\left(+1\left|\beta_{1},\right| 0\right\rangle\right)}}_{\bar{p}_{01}} . \\
p_{11} \leq \underbrace{\frac{\left.P_{A B}\left(-1+1 \mid 0 \beta_{1}, \rho_{\exp }\right)-P_{B}\left(+1\left|\beta_{1},\right| 0\right\rangle\right) P_{A}\left(-1 \mid 0, \rho_{\exp }\right)}{\left.\left.P_{B}\left(+1\left|\beta_{1},\right| 1\right\rangle\right)-P_{B}\left(+1\left|\beta_{1},\right| 0\right\rangle\right)}}_{\bar{p}_{11}} .
\end{gathered}
$$

(S.16)

Eqs. (S.13)-(S.14)-(S.15)-(S.16) allows one to bound the maximal value that $W$ can take on the set of two-qubit separable states through Eq. (S.11). In the next section, we will show how to extend this entanglement witness to qudits, i.e. to the case where the modes A and B are potentially filled with more than one photon. Even if the bounds previously derived hold in the qudit case, we will need a bound on the probability $p_{n \geq 2 B}$ to find strictly more than 1 photon in $B$. Such a bound can be obtained using the displacement amplitude $\beta_{2}(\sim 2.64)$ such that $\left.\left.P_{B}\left(+1\left|\beta_{2},\right| 0\right\rangle\right)=P_{B}\left(+1\left|\beta_{2},\right| 1\right\rangle\right)\left(\right.$ and $\left.P_{B}\left(+1\left|\beta_{2},\right| n \geq 2\right\rangle\right)<$ $\left.\left.P_{B}\left(+1\left|\beta_{2},\right| 0\right\rangle\right)\right)$. In particular, we have

$$
\begin{aligned}
p_{n \geq 2 B} & =\sum_{n \geq 2} \operatorname{tr}\left(\rho_{\exp }|n\rangle\left\langle\left. n\right|_{B}\right)\right. \\
& \leq \underbrace{\frac{\left.P_{B}\left(+1 \mid \beta_{2}, \rho_{\exp }\right)-P_{B}\left(+1\left|\beta_{2},\right| 0\right\rangle\right)}{\left.\left.P_{B}\left(+1\left|\beta_{2},\right| 3\right\rangle\right)-P_{B}\left(+1\left|\beta_{2},\right| 0\right\rangle\right)}}_{p_{B}^{*}} .
\end{aligned}
$$

Note that in mode A, $p_{n \geq 2 A}$ can be bounded from an autocorrelation measurement as shown already in Ref. [3]. We here give the basic idea to make this manuscript self-content. An autocorrelation measurement uses a 50/50 beam-splitter followed by two detectors, one for each output path. Labelling $p_{n A}$ the probability to find $n$ photons in A exactly, the probability $p_{\text {coinc }}$ to get a two-fold coincidence after the beam-splitter is given by $p_{\text {coinc }}=\sum_{n \geq 2} \frac{n}{2^{n}}\left(2^{n-1}-1\right) p_{n A} \geq \frac{1}{2} \sum_{n \geq 2} p_{n A}=\frac{1}{2} p_{n \geq 2 A}$. Therefore, $p_{n \geq 2 A} \leq \underbrace{2 * p_{\text {coinc }}}_{p_{A}^{*}}$.

\section{SUPPLEMENTAL MATERIAL IV}

Now consider the case where the state has an arbitrary dimension in the Fock space. Such a state can be written as

$$
\rho=\left(\begin{array}{cc}
\rho_{n_{a} \leq 1 \cap n_{b} \leq 1} & \rho_{\text {coh }} \\
\rho_{\text {coh }}^{\dagger} & \rho_{n_{a} \geq 2 \cup n_{b} \geq 2}
\end{array}\right) .
$$

By linearity of the trace, we have

$$
\begin{aligned}
\operatorname{tr}(W \rho) & =\operatorname{tr}\left(\rho_{n_{a} \leq 1 \cap n_{b} \leq 1} W\right)+\operatorname{tr}\left(\left(\rho_{c o h}^{\dagger}+\rho_{c o h}\right) W\right) \\
& +\operatorname{tr}\left(\rho_{n_{a} \geq 2 \cup n_{b} \geq 2} W\right) .
\end{aligned}
$$

Let us treat those terms one by one. The first term is the subject of Sec. 2, where we showed that $\operatorname{tr}\left(W \rho_{n_{a} \leq 1 \cap n_{b} \leq 1}\right) \leq W_{\text {ppt }}(\vec{p})$ (see Eq. (S.11)) and where the elements of $\vec{p}$ are bounded by the expressions given in Sec. 3.

To bound the second term, let us recall that $W$ does not contain coherences between sectors of different total photon number. Therefore,

$$
\operatorname{tr}\left(\left(\rho_{\text {coh }}^{\dagger}+\rho_{\text {coh }}\right) W\right) \leq 2\left(\left|C_{11}^{20} W_{11}^{20}\right|+\left|C_{11}^{02} W_{11}^{02}\right|\right),
$$


where $C_{i j}^{k l}=\langle i j|\rho| k l\rangle$ and $W_{i j}^{k l}=\langle i j|W| k l\rangle$ are the off-diagonal terms of $\rho$ and $W$, respectively. The positivity of the state $\rho$ restricted to the subspace $\{|20\rangle,|02\rangle,|11\rangle\}$ implies $C_{11}^{k l} \leq \sqrt{p_{11} p_{k l}}$. Since $p_{20} \leq p_{A}^{*}, p_{02} \leq p_{B}^{*}$, and $p_{11} \leq \bar{p}_{11}$ we have

$$
\operatorname{tr}\left(\left(\rho_{c o h}^{\dagger}+\rho_{c o h}\right) W\right) \leq 2 \sqrt{\bar{p}_{11}}\left(\left|W_{11}^{20}\right| \sqrt{p_{A}^{*}}+\left|W_{11}^{02}\right| \sqrt{p_{B}^{*}}\right) .
$$

The maximum algebraic value of $W$ is equal to 1 , in such a way that the third term is upperbounded by $\operatorname{tr}\left(\rho_{n_{a} \geq 2 \cup n_{b} \geq 2} W\right) \leq$ $\operatorname{tr}\left(\rho_{n_{a} \geq 2 \cup n_{b} \geq 2}\right) \leq p_{A}^{*}+p_{B}^{*}=p^{*}$.

Finally, any state $\rho$, such that its restriction $\rho_{n_{a} \leq 1 \cap n_{b} \leq 1}$ remains positive under partial transpose, satisfies

$$
\begin{aligned}
\operatorname{tr}(W \rho) \leq W_{\mathrm{PPT}} & =W_{\mathrm{ppt}}(\vec{p}) \\
& +2 \sqrt{\bar{p}_{11}}\left(\left|W_{11}^{20}\right| \sqrt{p_{A}^{*}}+\left|W_{11}^{02}\right| \sqrt{p_{B}^{*}}\right) \\
& +p^{*} .
\end{aligned}
$$

Any state $\rho_{\text {exp }}$ such that $\operatorname{tr}\left(W \rho_{\text {exp }}\right)-W_{\text {PPT }}>0$ is necessary entangled in the qubit subspace, i.e. in the subspace with at most one photon locally.

\section{SUPPLEMENTAL MATERIAL V}

In this section, we compute the value that the witness takes in the setup presented in Fig. 2 of the main text when the single photons are produced from a spontaneous parametric down conversion source. Let us first describe the state of Fig. 2 (main text) in which the right mode $B$ of the source is heralded by a click in the detector on the left mode $D$ (corresponding bosonic operators $d, d^{\dagger}$ ). The output of the parametric down conversion source is a two mode squeezed state given by

$$
|\Psi\rangle_{\mathrm{SPDC}}=\sqrt{\frac{1}{1-T_{g}^{2}}} \sum_{k} T_{g}^{k}|k, k\rangle_{b, d},
$$

with the gain parameter $g$ and $T_{g}=\tanh (g)$. The non photon number resolving detector used to announce the creation of a photon pair is in our case a threshold detector with $\theta=1$ and efficiency $\eta_{h}=1-R_{h}$. Accordingly to Eq. (S.5) the operator corresponding to a such a detection event reads

$$
P_{\mathrm{s}}^{1, \eta_{h}}=\mathbb{1}-P_{\mathrm{ns}}^{1, \eta_{h}}=\mathbb{1}-R_{h}^{d^{\dagger} d} .
$$

Hence, a click in the mode $D$ prepares the mode $b$ in the state

$$
\begin{aligned}
\rho_{h} & \propto \operatorname{tr}_{d}\left(P_{\mathrm{s}}^{1, \eta_{h}}|\Psi\rangle_{\mathrm{SPDC}}\langle\Psi|\right) \\
& =\frac{1}{1-T_{g}^{2}}\left(\sum_{k}\left(T_{g}^{2}\right)^{k}|k\rangle_{b}\left\langle k\left|-\sum_{k}\left(T_{g}^{2} R_{h}\right)^{k}\right| k\right\rangle_{b}\langle k|\right) .
\end{aligned}
$$

One already notes that this expression corresponds to a difference between two thermal states. After proper normalization the state reads

$$
\begin{array}{r}
\rho_{h}=\frac{1-R_{h}^{2} T_{g}^{2}}{T_{g}^{2}\left(1-R_{h}^{2}\right)}\left[\rho_{\text {th }}\left(\bar{n}=\frac{T_{g}^{2}}{1-T_{g}^{2}}\right)\right. \\
\left.-\frac{1-T_{g}^{2}}{1-R_{h}^{2} T_{g}^{2}} \rho_{\text {th }}\left(\bar{n}=\frac{R_{h}^{2} T_{g}^{2}}{1-R_{h}^{2} T_{g}^{2}}\right)\right],
\end{array}
$$

where $\rho_{\text {th }}(\bar{n})=\frac{1}{1+\bar{n}} \sum_{k}\left(\frac{\bar{n}}{1+\bar{n}}\right)^{k}|k\rangle_{b}\langle k|$ is a thermal state with mean photon number $\bar{n}$.
Next, the state $\rho_{h}$ is sent on a beamsplitter with transmission $T$ going to Bob and reflection $R$ going to Alice. We label the beamsplitter transformation $U_{B S}|0\rangle_{a}=u_{\mathrm{BS}}^{T, R}=$ $e^{\tan \bar{\gamma} b a^{\dagger}} e^{\ln (\cos \bar{\gamma}) b^{+} b}|0\rangle_{a}$, where $\cos ^{2} \bar{\gamma}=T$ and $|0\rangle_{a}$ is the vacuum state of the empty input mode A.

The decomposition of $\rho_{h}$ in thermal states (S.25) allows one to compute the observed value of the witness (S.9)

$$
\langle W\rangle=\operatorname{tr}\left(W u_{\mathrm{BS}}^{T, R} \rho_{h} u_{\mathrm{BS}}^{T, R+}\right)
$$

from the value that the witness takes on a thermal state $W_{\text {th }}(\bar{n}) \equiv \operatorname{tr}\left(W u_{\mathrm{BS}}^{T, R} \rho_{\mathrm{th}}(\bar{n}) u_{\mathrm{BS}}^{T, R+}\right)$. Since the two-mode state $u_{\mathrm{BS}}^{T, R} \rho_{\mathrm{th}}(\bar{n}) u_{\mathrm{BS}}^{T, R+}$ shared by Alice and Bob is invariant under global phase rotations $U_{\varphi} \otimes U_{\varphi}$, it follows from Eq. (S.9) that the value of $W$ they observe is given by

$$
W_{\mathrm{th}}(\bar{n}) \equiv \operatorname{tr}\left(\sigma_{\bar{\beta}}^{7, \eta_{b}} \sigma_{\bar{\alpha}}^{1, \eta_{a}} u_{\mathrm{BS}}^{T, R} \rho_{\mathrm{th}}(\bar{n}) u_{\mathrm{BS}}^{T, R+}\right)
$$

We stress that in the equation above $\eta_{a}=\eta_{t} \eta_{D_{A}}$ and $\eta_{a}=\eta_{t} \eta_{D_{B}}$ are global efficiencies (with $\eta_{t}$ - source to detector transmission and $\eta_{D}$ - efficiency of the corresponding detector), and $\bar{\alpha}$ and $\bar{\beta}$ are the displacement amplitudes assuming that all the losses happen after the displacements.

From the $P$-representation of a thermal state and the transformation of a coherent state on a beamsplitter, we find

$$
\begin{array}{r}
u_{\mathrm{BS}}^{T, R} \rho_{\mathrm{th}}(\bar{n}) u_{\mathrm{BS}}^{T, R+}=\frac{1}{\pi \bar{n}} \int e^{-\frac{|\alpha|^{2}}{\bar{n}}} u_{\mathrm{BS}}^{T, R}|\alpha\rangle\langle\alpha| u_{\mathrm{BS}}^{T, R+} d^{2} \alpha \\
=\frac{1}{\pi \bar{n}} \int e^{-\frac{|\alpha|^{2}}{\bar{n}}}|\sqrt{T} \alpha, \sqrt{R} \alpha\rangle\langle\sqrt{T} \alpha, \sqrt{R} \alpha| d^{2} \alpha .
\end{array}
$$

Using Eqs. (S.7) and (S.28), we can rewrite Eq. (S.27) as

$$
\begin{aligned}
& W_{\text {th }}(\bar{n})=\frac{\eta_{b}^{7}}{6 !} \frac{d^{6}}{d\left(1-\eta_{b}\right)^{6}} \frac{1}{\eta_{b}}\left[1+4 \frac{e^{-\eta_{a}|\bar{\alpha}|^{2}-\eta_{b}|\bar{\beta}|^{2}+\frac{\bar{n}\left|\bar{\alpha} \eta_{a} \sqrt{R}+\bar{\beta} \eta_{b} \sqrt{T}\right|^{2}}{\bar{n}\left(\eta_{a} R+T \eta_{b}\right)+1}}}{\bar{n}\left(\eta_{a} R+T \eta_{b}\right)+1}\right. \\
& \left.-2 \frac{e^{-\frac{\eta_{a}|\bar{a}|^{2}}{\eta_{a} \bar{n} R+1}}}{\eta_{a} \bar{n} R+1}-2 \frac{e^{-\frac{\eta_{b}|\bar{\beta}|^{2}}{\eta_{b} \bar{n} T+1}}}{\eta_{b} \bar{n} T+1}\right]
\end{aligned}
$$

Finally, from the previous expression together with Eq. (S.25), we find

$$
\begin{aligned}
\langle W\rangle= & \frac{1-R_{h}^{2} T_{g}^{2}}{T_{g}^{2}\left(1-R_{h}^{2}\right)}\left[W_{\text {th }}\left(\frac{T_{g}^{2}}{1-T_{g}^{2}}\right)\right. \\
& \left.-\frac{1-T_{g}^{2}}{1-R_{h}^{2} T_{g}^{2}} W_{\text {th }}\left(\frac{R_{h}^{2} T_{g}^{2}}{1-R_{h}^{2} T_{g}^{2}}\right)\right] .
\end{aligned}
$$

As we already mentioned, to simplify the derivation we introduced here the displacement amplitudes $\bar{\alpha}$ and $\bar{\beta}$ assuming that the displacements happen before the losses $\eta_{a}$ and $\eta_{b}$. All along the main text, we use the notation $\alpha$ and $\beta$ which corresponds to the case where the displacements happen after the losses. The two quantities are of course simply related by $\alpha=\bar{\alpha} \eta_{a}$ and $\beta=\bar{\beta} \eta_{b}$. In practice the situation is yet different. The physical displacement $\alpha_{\text {exp }}$ and $\beta_{\text {exp }}$ in Fig. 2 of the main text happen after the transmission losses but before the detector inefficiencies. Those amplitudes are related to the notation used in this section by $\alpha_{\exp }=\bar{\alpha} \eta_{t}$ and $\beta_{\exp }=\bar{\beta} \eta_{t}$. 


\section{REFERENCES}

1. P. Sekatski, B. Sanguinetti, E. Pomarico, N. Gisin, and C. Simon, Phys. Rev. A 82, 053814 (2010).

2. A. Peres, Phys. Rev. Lett. 77, 1413 (1996).

3. F. Monteiro, V. Caprara Vivoli, T. Guerreiro, A. Martin, J.-D. Bancal, H. Zbinden, R. T. Thew, and N. Sangouard, Phys. Rev. Lett. 114, 170504 (2015).

4. M. J. Collet, Phys. Rev. A 38, 2233 (1988). 